\title{
SIRT5, functions in cellular metabolism with a multiple enzymatic activities
}

\author{
YANG Xin ${ }^{1}$, LIU BoYa ${ }^{1}$, ZHU WeiGuo ${ }^{2}$ \& LUO JianYuan ${ }^{1,3 *}$ \\ ${ }^{1}$ Department of Medical Genetics, Peking University Health Science Center, Beijing 100191, China; \\ ${ }^{2}$ Department of Biochemistry, Peking University Health Science Center, Beijing 100191, China; \\ ${ }^{3}$ Department of Medical \& Research Technology, School of Medicine, University of Maryland, Baltimore 21201, USA
}

Received May 23, 2015; accepted June 6, 2015; published online July 23, 2015

Citation: $\quad$ Yang X, Liu BY, Zhu WG, Luo JY. SIRT5, functions in cellular metabolism with a multiple enzymatic activities. Sci China Life Sci, 2015, 58: 912-914, doi: 10.1007/s11427-015-4902-8

Mammalian Sirtuins are the homologs of yeast Saccharomyces cerevisiae Sir2 (silent information regulator 2), which functions in chromatin silencing to prevent genomic instability and aging by catalyzing the histone deacetylation [1]. There are seven members in Sirtuin family (SIRT1-SIRT7). They all contain evolutionary conserved enzymatic domain which is nicotinamide adenine dinucleotide (NAD)-dependent histone deacetylase that targets a wide variety of histone or non-histone proteins for biological functions. Each of the Sirtuins has distinctive functions and cellular localization. SIRT1, $6 \& 7$ are localized in nucleus, and SIRT2 is localized at cytoplasm while SIRT3, 4 $\& 5$ are localized in mitochondria [2].

SIRT5 is a unique member among the Sirtuin family because it contains multiple enzymatic activities. It was initially reported as a NAD-dependent histone deacetylase [3], More recently, SIRT5 has been found that it also contains a NAD-dependent protein lysine demalonylase and desuccinylase activities [4]; and lysine glutarylase activity [5]. The multiple enzymatic activities of SIRT5 indicate that SIRT5 is a multiple functional proteins in its regulation of cellular metabolism. Here, we will discuss how multiple enzymatic activities of SIRT5 in regulation of cellular metabolism.

SIRT5 is primarily defined as a lysine deacetylase in mitochondria which can deacetylate carbamoyl phosphate

*Corresponding author (email: jluo@ som.umaryland.edu) synthetase 1 (CPS1), a rating-limiting enzyme in the first step of urea cycle [3]. In vivo test in cultured primary hepatocytes and in mice showed that CPS1 is regulated by SIRT5 directly through deacetylation under calorie restriction environment. Although SIRT5 knockout (KO) mice do not show remarkable phenotypes and diseases, its blood ammonia is significant higher than wide-type after 48 $\mathrm{h}$ fasting. SIRT5 overexpressing transgenic (Sirt5-Tg) mice were also created to confirm its relationship with CPS1. CPS1 activity and urea production in Sirt5-Tg mice are higher than wide-type mice [6], indicating that SIRT5 deacetylates CPS1 to activate its function in urea cycle for generating more urea. Urea cycle is tightly regulated by SIRT5 despite ornithine transcarbamoylase (OTC) and other enzymes in this cycle are not targeted by SIRT5. Another SIRT5 target is Urate oxidase (UOX). SIRT5 deacetylates UOX and activates its enzymatic activity in Sirt5-Tg mice. Furthermore, SIRT5 has also been found to deacetylate cytochome $\mathrm{c}$, which located in the mitochondrial intermembrane space as the core protein of oxidative metabolism. Other studies showed that under ethanol stress SIRT5 reduced its deacetylase activity in hepatic cells.

Through structural analysis, Lin and colleagues [4] found that the acyl peptide binds SIRT5 better than the acetyl peptide, and these acyl peptides contain succinyl peptide and malonyl peptide. These findings led to identify that SIRT5 contains a desuccinylase and demalonylase activities. Other members of Sirtuins family have no activity on acyl peptide, 
which makes SIRT5 a unique protein among the family members. The mechanism for desuccinylation and demalonylation catalyzed by SIRT5 is the same to mechanism for deacetylation catalyzed by Sirtuins. By LC-MS/MS, several succinylated proteins were identified: 3-hydroxy-3-methylglutaryl-CoA synthase 2, thiosulfate sulfurtransferase, and aspartate aminotransferase.

Anti-Kmal antibody is generated for detecting global malonylation in Escherichia coli and Hela cells. With the help from it, many proteins have been identified that they could be malonylated. These findings established malonylation as a new type of protein post-translational modification. And SIRT5 was identified as a lysine demalonylating enzyme which can catalyze the demalonylation process both in vitro and in vivo [7].

Through examination of proteins from mouse embryo fibroblasts (MEFs) and mouse liver cells by stable isotope labeling with amino acids (SILAC), the global lysine succinylation in mammals was defined [8]. The number of overlapped Ksuc sites between MEFs and mouse liver cells is only 294 , indicating that the great differences in the succinylome between cultured cells and mouse tissue are exist. Furthermore, there are only 282 sites overlapped between lysine succinylation sites and lysine acetylation sites. SIRT3 is thought to play the important role in regulating overall lysine acetylation in mitochondria. This data suggested that a numbers of proteins are targeted by SIRT5 and SIRT3 at the same time. SIRT5 deficiency resulted in increased lysine succinylation, however, global lysine acetylation was impacted weakly. Interestingly, SIRT5 was also found localized outside mitochondria, This led to speculating that SIRT5 may play a role in regulating chromatin function with histone proteins. STRING database was used to validate the protein-protein interaction networks of succinylome. HMGB1-HMGB2-HSC70-ERP60-GAPDH complex, the Pyruvate Dehydrogenase Complex (PDC) and the SDH-mABC1-PIC-ANT-ATPase complex are among the most convincing complex, suggesting that the SIRT5 enzymatic activities play an important role in regulating cellular processes. They also examined the process of succinylation mechanism using 3-NPA and malonate (the inhibitors of $\mathrm{SDH}$ ) in succinate dehydrogenase complex subunit $\mathrm{C}$ (SDHC) knockdown mice. The outcome showed that succinylation levels of the proteins were slight, which suggested that the mechanism of succinylation is still unclear.

Normal and KO SIRT5 mice were examined for the global lysine succinylation by Verdin group [9]. Hypersuccinylation of mitochondria proteins was found in SIRT5 KO mice. They found the conservation of SIRT5-targeted succinylation sites among 6 species (human, rat, cattle, bird, frog, and zebra fish). By examining the isolated hepatocytes from wild type (WT) and SIRT5 KO mice, they found the obvious decreased fatty acid oxidation and increased accumulation of acylcarnitines in SIRT5 KO mice. Ketone body production is also decreased and connected directly to the hypersuccinylation of HMGCS2. Kinetic analysis revealed that SIRT5 regulates 3-hydroxy-3-methylglutaryl-CoA synthase 2 (HMGSC2) desuccinylation at K83, K310 to restore the binding pocket for phosphate groups of acetyl-CoA to accumulate ketone body production. Meanwhile, HMGSC2 deacetylation was regulated by SIRT3 other than SIRT5. Cu/Zn superoxide dismutase (SOD1) may be another SIRT5 target which can be desuccinylated so as to eliminate reactive oxygen species (ROS) .

SIRT5 was also regulated by other proteins. Peroxisome proliferator-activated receptor $\gamma$ coactivator 1- $\alpha$ (PGC-1 $\alpha$ ) and AMP-activated protein kinase (AMPK) were identified to regulate SIRT5 in mRNA level and led to change of the global succinylation level.

SIRT5 was also found that it can regulate lysine glutarylation modification by mass spectrometry recently. The glutarylome has been widely analyzed and revealed that 191 proteins contains 683 glutarylaion sites [5], indicating that glutarylaion is high enriched in mitochondrial and cellular metabolism. In vitro deglutarylation reaction revealed that nicotinamide can inhibit the glutarylase activity of SIRT5, and Sirtinol, trichostatin A (TSA), NaBu have no effect on SIRT5 glutarylase activity. In contrast, they also found that both SIRT3 and SIRT4 have no glutarylation function at all.

CPS1 is an important target of SIRT5 for glutarylation. The latest research revealed that SIRT5 knockdown mouse showed universal hyperglutarylation feature and CPS1 is hyperglutarylated in SIRT5 knockdown mouse after fasting. SIRT5 KO mouse also showed increased sensitivity to dietary changes. The glutaryl-CoA dehydrogenase (GCDH) is the key protein to the human Glutaric Acidemia I disease. Interestingly, GCDH KO mice expressed higher levels of glutaryl-CoA suggesting that the glutaryl-CoA leads to glutarylation modification directly. Comparing to WT mice, the GCDH KO mice expressed more CPS1 protein to make up of the decreased CPS1 protein enzymatic activity. These results indicate that cells can maintain its glutarylation activity and protein level balance by internal regulation.

The majority of SIRT5 protein exists in mitochondrial. However, it was also found in other compartments of the cell. The relationship with histone proteins and coexpression with SIRT3 in nucleus indicate it may play broad role in regulating cellular functions. Global succinylation and glutarylation revealed that SIRT5 may also regulate its substrates outside mitochondrial.

Recent findings revealed that SIRT3 plays the major role in protein deacetylaion in mitochondria rather than SIRT4 and SIRT5. In SIRT4 and SIRT5 KO mice, the mitochondrial overall acetylation levels do not change compared to wide-type mice [10]. In contrast, the obvious global hyperacetylation was found in SIRT3 KO mice, indicating its major status as a mitochondrial deacetylase. As for the SIRT5 catalytic efficiency of deacetylation, it is much less compared to demalonylation and desuccinylation activities (almost 1,000 fold). This result suggests that SIRT5 regu- 
lates protein functions through demalonylation, desuccinylation and glutarylation other than deacetylation.

The mRNA levels of many proteins in mitochondrial are not greatly changed in SIRT5 $\mathrm{KO}$ mice, indicating the internal regulation in cellular metabolism. It can be inferred that if activity or quantity of one enzyme is decreased, the other enzymes can be regulated up or down to cope with this change. This phenomenon is obvious in some core metabolism such as tricarboxylic acid (TCA) cycle and amino acid metabolism. The downstream production can be influenced by the upstream production and enzyme activity. Although SIRT5 has been found playing multiple functions in regulating cellular metabolism (Figure 1), future work still needs to be focused on its direct substrates and functions in regulating biological processes.

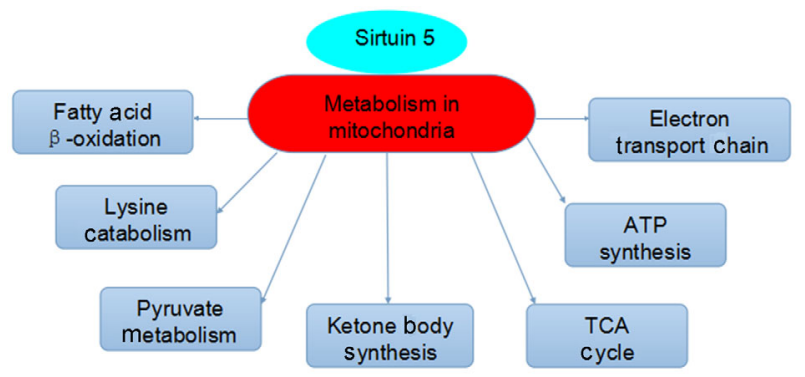

Figure 1 Identified metabolism pathways regulated by SIRT5.

1 Imai S, Armstrong CM, Kaeberlein M, Guarente L. Transcriptional silencing and longevity protein Sir2 is an NAD-dependent histone deacetylase. Nature, 2000, 403: 795-800

2 Haigis MC, Sinclair DA. Mammalian sirtuins: biological insights and disease relevance. Annu Rev Pathol, 2010, 5: 253-295
3 Nakagawa T, Lomb DJ, Haigis MC, Guarente L. SIRT5 Deacetylates carbamoyl phosphate synthetase 1 and regulates the urea cycle. Cell, 2009, 137: 560-570

4 Du J, Zhou Y, Su X, Yu JJ, Khan S, Jiang H, Kim J, Woo J, Kim JH, Choi BH, He B, Chen W, Zhang S, Cerione RA, Auwerx J, Hao Q, Lin H. Sirt5 is a NAD-dependent protein lysine demalonylase and desuccinylase. Science, 2011, 334: 806-809

5 Tan M, Peng C, Anderson KA, Chhoy P, Xie Z, Dai L, Park J, Chen Y, Huang H, Zhang Y, Ro J, Wagner GR, Green MF, Madsen AS, Schmiesing J, Peterson BS, Xu G, Ilkayeva OR, Muehlbauer MJ, Braulke T, Mühlhausen C, Backos DS, Olsen CA, McGuire PJ, Pletcher SD, Lombard DB, Hirschey MD, Zhao Y. Lysine glutarylation is a protein posttranslational modification regulated by SIRT5. Cell Metab, 2014, 19: 605-617

6 Ogura M, Nakamura Y, Tanaka D, Zhuang X, Fujita Y, Obara A, Hamasaki A, Hosokawa M, Inagaki N. Overexpression of SIRT5 confirms its involvement in deacetylation and activation of carbamoyl phosphate synthetase 1. Biochem Biophys Res Commun, 2010, 393: 73-78

7 Peng C, Lu Z, Xie Z, Cheng Z, Chen Y, Tan M, Luo H, Zhang Y, He W, Yang K, Zwaans BM, Tishkoff D, Ho L, Lombard D, He TC, Dai J, Verdin E, Ye Y, Zhao Y. The first identification of lysine malonylation substrates and its regulatory enzyme. Mol Cell Proteomics, 2011, 10: M111.012658

8 Park J, Chen Y, Tishkoff DX, Peng C, Tan M, Dai L, Xie Z, Zhang Y, Zwaans BM, Skinner ME, Lombard DB, Zhao Y. SIRT5-mediated lysine desuccinylation impacts diverse metabolic pathways. Mol Cell, 2013, 50: 919-930

9 Rardin MJ, He W, Nishida Y, Newman JC, Carrico C, Danielson SR, Guo A, Gut P, Sahu AK, Li B, Uppala R, Fitch M, Riiff T, Zhu L, Zhou J, Mulhern D, Stevens RD, Ilkayeva OR, Newgard CB, Jacobson MP, Hellerstein M, Goetzman ES, Gibson BW, Verdin E. SIRT5 regulates the mitochondrial lysine succinylome and metabolic networks. Cell Metab, 2013, 18: 920-933

10 Lombard DB, Alt FW, Cheng HL, Bunkenborg J, Streeper RS, Mostoslavsky R, Kim J, Yancopoulos G, Valenzuela D, Murphy A, Yang Y, Chen Y, Hirschey MD, Bronson RT, Haigis M, Guarente LP, Farese RV Jr, Weissman S, Verdin E, Schwer B. Mammalian Sir2 homolog SIRT3 regulates global mitochondrial lysine acetylation. Mol Cell Biol, 2007, 27: 8807-8814

Open Access This article is distributed under the terms of the Creative Commons Attribution License which permits any use, distribution, and reproduction in any medium, provided the original author(s) and source are credited. 